\title{
Inter-harmonics in multi-terminal VSC-based HVDC systems
}

\author{
Minxiao HAN ${ }^{1}$, Phuchuy NGUYEN ${ }^{1}$, Wenli YAN $^{2}$
}

\begin{abstract}
Multi-terminal voltage sourced converter (VSC)-based high voltage direct current (HVDC) system composes of a number of VSCs connected to an HVDC grid (or dc grid). The dc grid is often configured by cable interconnections between converter stations, thus imposing resonance issues affecting harmonic interaction in the system. Based on the harmonic transfer characteristics of VSC and HVDC systems, the appearance and the interaction of inter-harmonics in the multi-terminal VSC-based HVDC system are analyzed. Simulation models are built and implemented using SimPowerSystems in MATLAB. The simulation results show that, a series of inter-harmonics are produced and tend to be dominant in low-frequency range. Especially in the dc grid, the inter-harmonic transfer can be magnified due to inter-harmonic resonances. The complex resonance issues in the dc grid are investigated in combination with interaction through the VSC, it is beneficial to harmonic filter designs as well as other harmonic mitigation methods.
\end{abstract}

CrossCheck date: 14 April 2015

Received: 12 July 2014/Accepted: 15 April 2015/Published online: 15 December 2015

(C) The Author(s) 2015. This article is published with open access at Springerlink.com

$\square$ Phuchuy NGUYEN

huynp@epu.edu.vn

Minxiao HAN

hanminxiao@263.net

Wenli YAN

yanwenli11@163.com

1 School of Electrical Engineering, North China Electric Power University, Beijing 102206, China

2 School of Mathematical \& Physical Science School, North China Electric Power University, Beijing 102206, China
Keywords Voltage sourced converter, Inter-harmonic, VSC-based HVDC, Multi-terminal VSC-based HVDC, DC-resonance

\section{Introduction}

The VSC-based HVDC systems have been utilized in power system for many years since the first test in 1997. For emerging applications involving the integration of large scale wind power plants, the multi-terminal VSCbased HVDC systems win over the conventional LCCbased HVDC systems. This is due to the VSC-based HVDC has characteristics as independent control of active and reactive power, possibility to supply passive weak networks and black-start capability, no commutation failure, and reverse power transmission without reversing voltage polarity [1-3].

The VSC is a main component of a VSC-based HVDC system, which generates harmonics on its both ac and dc sides. These harmonics are called characteristic harmonics of the VSC, which are directly associated with the type of VSC technologies, modulation techniques and the switching frequency [4]. The harmonic interaction between ac and dc sides of a VSC [5, 6] shows that, when the ac supply of an HVDC system is unbalanced or one side of the converter containing background harmonics, the both acand dc-sides of the VSC are produced as non-characteristic harmonics. The non-characteristic harmonics may be nonintegral and referred to inter-harmonics [7, 8]. The propagation of background harmonics from one end ac system to the remote one in a point-to-point VSC-based HVDC system has been studied in [9]; however, the harmonic interaction in a multi-terminal VSC-based HVDC system has not been investigated yet. 
In a multi-terminal VSC-based HVDC system, a number of possible topologies are established, thus affecting the appearance of parallel resonance points at low frequency range in the dc grid $[3,10]$. If that a modest harmonic current at the resonance frequency will cause a relative high dc harmonic voltage, correspondingly, it causes significant harmonic voltages on the ac sides. Moreover, the harmonic interaction at each converter station is not only affected by other harmonic interaction at the other converter stations in the system, and this should be investigated in more detail.

From the analysis of the harmonic transfer through the VSC, the origins of inter-harmonics in the ac systems and the dc grid of a multi-terminal VSC-based HVDC system are pointed out in this paper. Because the system has asynchronous HVDC connections whose ac grids differ from frequency, dc-side characteristic harmonics of one end converter will be the ripples to the other end converter. Consequently, these ripples could induce inter-harmonics in every ac system. Moreover, a background harmonic in one end ac system will be transferred to produce a series of inter-harmonics in the other end ac systems with different fundamental frequencies. The magnification of these interharmonics strongly depends on the operating configurations of the dc grid. One further important point to note is that the dc capacitors of the three-level NPC VSCs could cause low-frequency inter-harmonics to be produced in every ac system, especially the negative-sequence subharmonics, which have smaller frequencies than the system frequency. In all cases, the power contribution of all connected ac systems also has certain influence on the harmonic interaction in the entire system.

\section{Multi-terminal VSC-based HVDC system}

The schematic diagram of the multi-terminal VSCHVDC system for inter-harmonic characteristic study is illustrated in Fig. 1, in which the ac system 1 is a $60 \mathrm{~Hz}$ system and the other ac systems are $50 \mathrm{~Hz}$ systems.

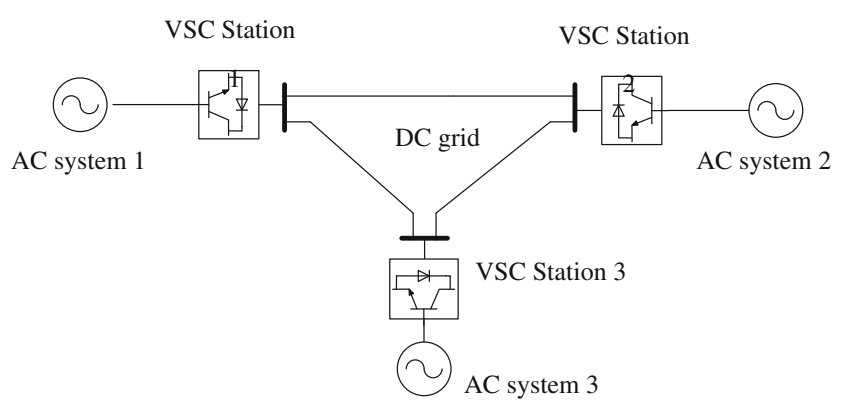

Fig. 1 Schematic diagram of the studied system
In each converter station, the converter is the type of three-level neutral point clamped (NPC); a three-phase smoothing converter reactor is placed between the converter and a converter transformer. The converter transformer is $\mathrm{Yn} / \mathrm{Y}$ connection type where the $\mathrm{Y}$ winding is connected to the reactor, resulting in decoupling the ac system from the triple harmonics produced by the converter. The ac shunt passive filter group is located between the converter transformer and the converter reactor, comprised of a high-pass filter to filter out the harmonics at frequencies higher than the carrier frequency and a double tuned filter to filter 5th and the harmonic at twice carrier frequency of the VSC. The dc-side of the VSC uses reservoir dc capacitors to equalize dc voltage, enhance the system dynamics and reduce the dc-side voltage ripple. The dc-side filter circuit consists of the 3rd order tuned filters and dc-smoothing reactors.

\section{Relationship between ac and dc quantities of VSC}

Figure 2 shows the phase quantities of a three-phase three-level NPC-VSC. Corresponding to three voltage levels, the switching function $k_{x}(t)$ of phase $x(x=\mathrm{a}, \mathrm{b}, \mathrm{c})$ takes the values 1,0 , and -1 which is relative to three switching states of the valves. For the VSC using pulsewidth modulation (PWM) technique, $k_{x}(t)=1$ is relative to the valve switched to the positive dc pole, corresponds to the positive half cycle modulation wave; $k_{x}(t)=-1$ is relative to the valve switched to the negative dc pole, corresponds to the negative half cycle modulation wave; and $k_{x}(t)=0$ is relative to the valve switched to the midpoint.

The relationship between ac and dc side quantities can be expressed as:

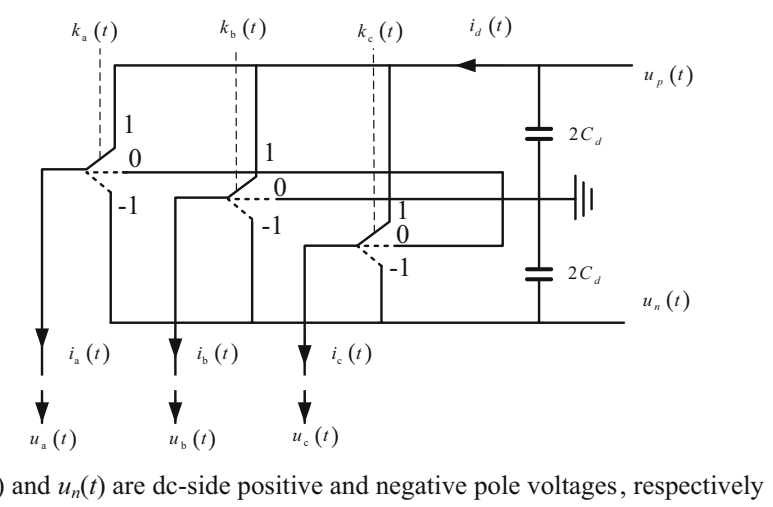

Fig. 2 Phase quantities of a three-level VSC 
$\left\{\begin{array}{l}u_{\mathrm{a}}(t)=k_{\mathrm{a}}(t) u_{\mathrm{a} d}(t) \\ u_{\mathrm{b}}(t)=k_{\mathrm{b}}(t) u_{\mathrm{b} d}(t) \\ u_{\mathrm{c}}(t)=k_{\mathrm{c}}(t) u_{\mathrm{c} d}(t)\end{array}\right.$

$i_{d}(t)=\frac{k_{\mathrm{a}}(t) i_{\mathrm{a}}(t)+k_{\mathrm{b}}(t) i_{\mathrm{b}}(t)+k_{\mathrm{c}}(t) i_{\mathrm{c}}(t)}{2}$

In (1), the dc voltage relates to the output voltage of phase $x$ can be defined as [9]:

$u_{x d}(t)=\left[u_{p}(t) k_{x p}(t)+u_{n}(t) k_{x n}(t)\right]$

where $k_{x p}(t)$ and $k_{x n}(t)$ are step functions corresponding to each half cycle of modulation wave of phase $x$, with characteristic of phase a, $k_{\mathrm{a} p}(t)-k_{\mathrm{a} n}(t)=1$ and $k_{\mathrm{a} p}(t)+$ $k_{\mathrm{a} n}(t)$ is defined as:

$k_{\mathrm{a} p}(t)+k_{\mathrm{a} n}(t)=\frac{4}{\pi} \sum_{h=1,3,5 \ldots}^{\infty} \frac{1}{h} \sin \frac{h \pi}{2} \cos \left(h \omega_{1} t\right)$

In the case of $u_{p}(t)=-u_{n}(t)=u_{d}(t) / 2$, the relationships between ac-and dc-side quantities of VSC, without the zero sequence components, can be determined in the general formula of the space vector [5]:

$\bar{u}_{V}(t)=\frac{\bar{K}(t) u_{d}(t)}{2}$

$i_{d}(t)=\frac{3}{4} \operatorname{Re}\left\{[\bar{K}(t)]^{*} \bar{i}_{V}(t)\right\}$

where $\bar{u}_{V}(t), \bar{i}_{V}(t)$ are space vectors of ac-side voltage and current; $u_{d}(t), i_{d}(t)$ are dc-side voltage and current; $\bar{K}(t)$ is space vector of three-sinusoidal switching functions.

The switching function of the three-level NPC-VSC is analyzed in [9] composed of the largest fundamental switching component and the other high-order switching components (see Appendix A). The space vector representation of any $N$ th switching component has the form as:

$\bar{K}_{N}(t)=\hat{K}_{N} \mathrm{e}^{\mathrm{j} \omega_{N} t}$

As a fundamental switching component, $\hat{K}_{N}=M$ is the modulation index, and $\omega_{N}=\omega_{1}$ is the fundamental angular frequency.

\section{Origin of inter-harmonics}

\subsection{DC side inter-harmonics}

\subsubsection{DC side characteristic harmonics acting as inter- harmonics}

The studied system in the Fig. 1 forms asynchronous interconnections between different frequency ac systems. Therefore, the dc-side characteristic harmonics of VSC1, which are triple harmonics [11, 12], can act as the dc-side ripples and also are inter-harmonics source to the converters at the other two ends, i.e., VSC2 and VSC3. The same mechanisms are also applied to the dc-side characteristics of the VSC2 or VSC3 acting as the inter-harmonic sources to the VSC1. Consequently, the all three converters will operate to cause the different harmonics on both acand dc-sides.

\subsubsection{External impacts}

Assuming that the ac-side current of the VSC1 consists of harmonic components expressed in the space vector form as:

$\bar{i}_{V 1 h}(t)=\hat{i}_{1 h}^{+} \mathrm{e}^{\mathrm{j} \omega_{h} t}+\hat{i}_{1 h}^{-} \mathrm{e}^{-\mathrm{j} \omega_{h} t}$

The first part in (8) is the positive-sequence harmonic, and the second is the negative-sequence harmonic. Under the interaction of the $N$ th switching component, the dc positive pole current is written as:

$i_{d 1 h}(t)=\frac{3 \hat{K}_{N}}{4} \operatorname{Re}\left\{\hat{i}_{1 h}^{+} \mathrm{e}^{-\mathrm{j}\left(\omega_{N}-\omega_{h}\right) t}+\hat{i}_{1 h}^{-} \mathrm{e}^{-\mathrm{j}\left(\omega_{N}+\omega_{h}\right) t}\right\}$

Theoretically speaking, if the ac side of the VSC1 exists a positive-sequence harmonic, the dc side will produce a harmonic with the frequencies of $\left|\omega_{N}-\omega_{h}\right|$; and the ac side harmonic is a positive-sequence one, the dc side harmonic has the frequency of $\left|\omega_{N}+\omega_{h}\right|$. If the ac side of VSC1 has an unbalanced harmonic, it has both the positive- and negative-sequence components as in (8); therefore, there will be two side band harmonics on the dc-side. For example, considering the fundamental switching component, the dc side harmonics will have frequency of $\omega_{h}-\omega_{1}$ and $\omega_{h}+\omega_{1}$, respectively. They are inter-harmonics if harmonics in (8) are inter-harmonics.

\subsection{AC side inter-harmonics}

\subsubsection{External impacts}

The inter-harmonics on the ac side of the VSC may be those that propagated from distant areas in the connected ac systems or originated from the dc side harmonics.

The connected ac system may be inter-harmonic polluted by nonlinear connected loads including static frequency converters, cycloconverters, subsynchronous converter cascades, adjustable speed drives, arc furnaces; and of course including the loads not pulsating synchronously with the fundamental power system frequency [7, 8].

Background harmonics (or ripples) could exist on the dc side of a VSC-based HVDC and convey to the ac side. Assuming that the dc-side voltage comprises a background harmonic with angular frequency $\omega_{r}$ which is not an integer 
of the fundamental frequency, say the $\omega_{1}$, written in the form as:

$u_{d r}(t)=\hat{u}_{d r} \cos \left(\omega_{r} t\right)=\frac{\hat{u}_{d r}\left(\mathrm{e}^{\mathrm{j} \omega_{r} t}+\mathrm{e}^{-\mathrm{j} \omega_{r} t}\right)}{2}$

Under the interaction of the $N$ th switching component, the ac side voltage is

$\bar{u}_{V 1 r}(t)=\frac{\hat{K}_{N}}{4} \hat{u}_{d r}\left(\mathrm{e}^{\mathrm{j}\left(\omega_{r}+\omega_{N}\right) t}+\mathrm{e}^{-\mathrm{j}\left(\omega_{r}-\omega_{N}\right) t}\right)$

As can be seen that, the two side band inter-harmonics on the ac side will be $\left|\omega_{N} \pm \omega_{r}\right|$, and the sequence of the harmonics is the same to the sequence of the switching component for any $N>r$. For any $N<r$, such as the fundamental switching component $N=1<r$, one dc harmonic will cause a higher and a lower order harmonics on the ac side. The higher order harmonic has positive sequence and the lower one has negative sequence.

\subsubsection{DC capacitor ripples}

In the case of the three-level NPC VSC, the dc midpoint current contains a large third-order harmonic component, causing the 3rd-order harmonic voltages (the ripples) on the $\mathrm{dc}$ capacitors. These ripples in turn result in low-order voltage harmonics on the ac-side [11]. In the studied three-terminal VSC-based HVDC system, the midpoint current of one VSC could flow through the earth return paths to the other VSCs, causing the ripples on those VSCs' dc capacitors. Assuming that the ripple on the dc capacitor of the VSC2 caused by the midpoint current of the VSC1 is expressed as:

$u_{3 r}(t)=U_{3 r} \cos \left(3 \omega_{1} t+\xi\right)$

where $\omega_{1}$ is the fundamental frequency of the ac system 1; $\xi=\pi-\tan ^{-1}(1.5 \tan \gamma)$ with $\gamma$ is the phase delay of the currents with respect to the terminal voltages on the ac-side of the VSC1.

The dc positive and negative pole voltages at VSC2 can be written as:

$\left\{\begin{array}{l}u_{2 p}(t)=\frac{U_{d}}{2}+U_{3 r} \cos \left(3 \omega_{1} t+\xi\right) \\ u_{2 n}(t)=\frac{-U_{d}}{2}+U_{3 r} \cos \left(3 \omega_{1} t+\xi\right)\end{array}\right.$

Substituting (13) into (3) and then to (1), and if just considering the interaction of the fundamental switching component, the harmonic components of the ac output voltage of the VSC2, for example, the phase a output voltage can be calculated as:

$u_{a 2}(t)=\frac{M U_{3 r}}{\pi} \sum_{h=1,3,5 \ldots}^{\infty} \frac{1}{h} \sin \frac{h \pi}{2} \cos \left(\left[3 \omega_{1} \pm h \omega_{2} \pm \omega_{2}\right] t+\xi\right)$
From (14), because $\omega_{1} \neq \omega_{2}, \omega_{2}$ is the fundamental frequency of the ac system 2, the inter-harmonics will appear on the ac side of the VSC2 and may have smaller frequency then the fundamental, which could damage rotating machine connected to the system, especially in case of negative-sequence sub-harmonics.

\subsubsection{Unsymmetrical conditions}

When the point of common coupling (PCC) of the ac system 1 is subjected to single phase-to-ground fault, the HVDC system will operate under unbalanced conditions, and VSC is quite sensitive to the negative-sequence component in the ac voltage [13].

Based on the theory of symmetrical components, an unbalanced quantity comprises three balanced components of positive-, negative- and zero-sequence components. The space vector corresponding to three-phase current without the zero-sequence component is:

$\bar{i}_{1 s}(t)=\hat{I}_{1 s}^{+} \mathrm{e}^{\mathrm{j} \omega_{1} t}+\hat{I}_{1 s}^{-} \mathrm{e}^{-\mathrm{j}\left(\omega_{1} t+\varphi\right)}$

where $\hat{I}_{1 s}^{+}, \hat{I}_{1 s}^{-}$are amplitudes of positive- and negative-sequence components; $\varphi$ is phase-angle of the negative-sequence component, relative to the positive-sequence component.

Equation (15) has a similar form to (8) for unbalanced harmonics. As a result, a series of ripples will be induced on the dc-side of the converter where the component with frequency $2 \omega_{1}$ is dominant under the interaction of the fundamental switching component. Again, this dominant component is conveyed to the ac systems 2 and 3 to produce a series of inter-harmonics, where the negativesequence and positive-sequence components have frequencies at $2 \omega_{1}-\omega_{2}$ and $2 \omega_{1}+\omega_{2}$, respectively, are dominant.

\section{Harmonic interactions through dc grid}

Figure 3 illustrates the equivalent circuit of the dc grid in the three-terminal VSC-based HVDC system in Fig. 1 used for harmonic interaction study. In Fig. 3, to analyze the interaction of harmonics generated by the VSC1, the VSC1 is equivalent to harmonic current source looked from its dc side, the other VSCs may be considered with the same manner; $Z_{D 1}, Z_{D 2}, Z_{D 3}$ are equivalent impedances of the VSC and its ac side system, respectively for VSC1, VSC2 and VSC3; $Z_{12}, Z_{13}, Z_{23}$ are equivalent impedances of the dc cables; and $Z_{F 1}, Z_{F 2}, Z_{F 3}$ are respectively equivalent impedances of the dc filters respectively in three converter stations.

As can be seen that, the dc grid is a complex system where harmonic interaction of each converter station is not 


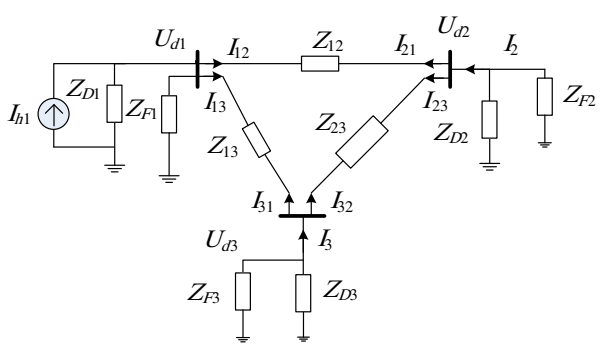

Fig. 3 Equivalent circuit of the dc grid

only affected by its self but also affected by other converter stations. At each node of the dc grid, the dc side filter design is implemented to avoid resonance at critical frequencies. However, the low-order resonant points still appear at non-integral frequencies which depend on a number of operating configurations of the grid. Therefore, the harmonic distortion at each node may be higher than those generated locally under certain circumstances, meaning that the ac side harmonic distortion increases as well. Moreover, the transferred power of each ac system can also contribute to the distribution of currents in the dc grid, affecting the harmonic interaction through the grid.

Figure 4 illustrates the impedance-frequency characteristics of the ac- and dc-side system looked from the output terminal of the converters. Clearly, different resonant points appear at low-frequency range, especially at $135 \mathrm{~Hz}$ at the dc terminal of the Stations 1 and 3, and at $115 \mathrm{~Hz}$ at the dc terminal of the Stations 2 and 3. If a harmonic appears at any resonant frequencies with any reasons, the harmonic will be magnified leading to harmonic

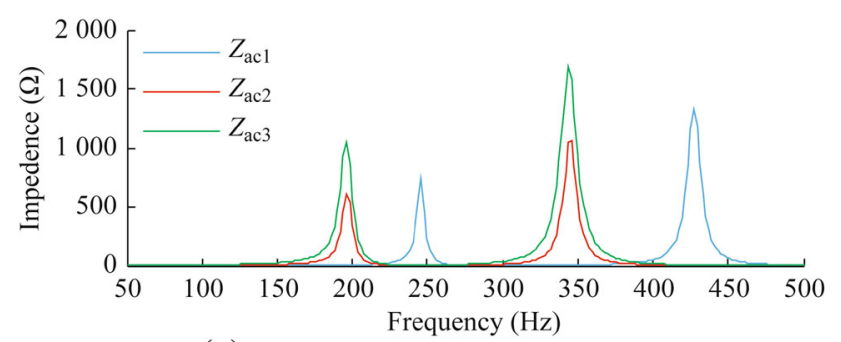

(a) AC side impedance-frequency characteristic

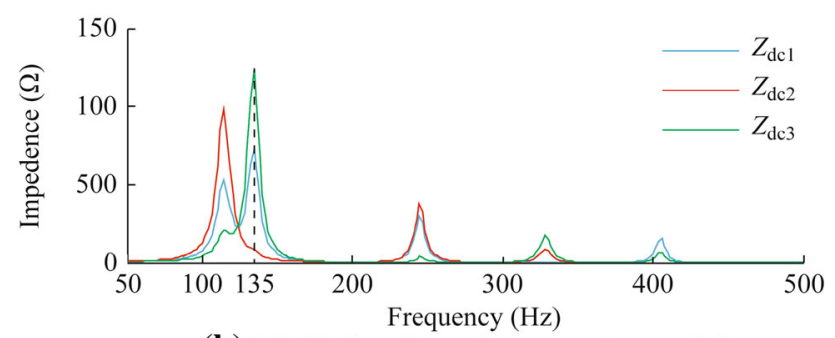

(b) DC side impedance-frequency characteristic

Fig. 4 Impedance-frequency characteristic of the system magnification in harmonic transferring through the grid or the converters.

\section{Simulation and result analysis}

Simulation models are set up for the VSC-based HVDC system in Fig. 1 using SimPowerSystems in MATLAB. The ac system 1 and ac system 3 support the ac system 2 through the dc grid with the power rate at $70 \%$ and $60 \%$ of their own capacity. In each model, the sending end VSCs are modeled to operate as power dispatcher while the receiving end VSC is modeled to operate as dc voltage regulator and reactive power controller [2]. In each VSC station, the main component, that is VSC, is a three-level NPC converter. The VSCs adopt the sinusoidal pulse width modulation (SPWM) technique. The frequency of the triangle carrier wave is 27 times of the fundamental frequency.

For acceptable accuracy of measurement of both harmonics and inter-harmonics, Fourier analysis is implemented with a number cycle windows chosen to get a suitable resolution spectrum for both two systems. For example, according to IEC-61000-4-7 standard, a 10 (for $50 \mathrm{~Hz}$ systems) or 12 (for $60 \mathrm{~Hz}$ systems) cycle windows is chosen, therefore a spectrum with $5 \mathrm{~Hz}$ resolution is achieved [7]. Due to the design of the systems with high pass filters on the ac sides, the high order harmonics could be suppressed. Moreover, the dc side impedance of the VSC has capacitive characteristic which is larger at higher frequency; therefore, the phase current and dc side voltage are just analyzed in low frequency range.

\subsection{Case 1: with background harmonics in the system}

The first case is the base-case giving a comparative view to the other cases. The spectra of phase current are shown in Fig. 5.

It can be seen that, the 5th-order harmonics in the current spectra are almost suppressed due to the double-tuned filters; the 7th-order harmonics appear in all three ac current spectra. These harmonics are caused by dc-capacitor ripples in the local converter stations. Because of the asynchronous interconnections, there was also a series of insignificant inter-harmonics in both phase current and $\mathrm{dc}$ side voltage (see Fig. 6).

Due to the effects of inter-harmonics, although the harmonic level of individual is within the limits defined by [14], the harmonic demand distortion (TDD) at the PCC1 of the ac system 1 is still higher than the acceptable value $(1.5 \%)$. 


\subsection{Case 2: dc capacitors affecting inter-harmonics}

In this case, the 3rd-order dc harmonic filters are removed from the model in Case 1. The inter-harmonics from the simulation agreed with the analytical results from (14) with the dominant inter-harmonics are $30 \mathrm{~Hz}$ and 270 $\mathrm{Hz}$ in the $60 \mathrm{~Hz}$ system (Fig. 7a); and $80 \mathrm{~Hz}$ in the $50 \mathrm{~Hz}$ system (Fig. 7b, c).

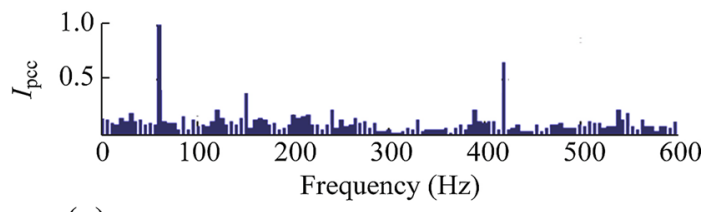

(a) $I_{\text {pcc }}$ of ac system 1 (\% of Load), TDD=2.3338 (\%)

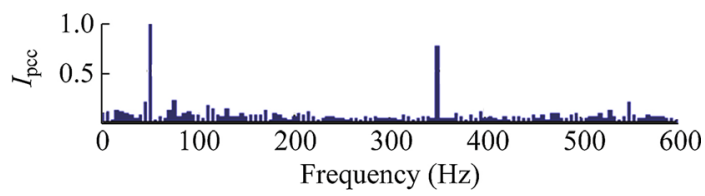

(b) $I_{\text {pcc }}$ of ac system 2 (\% of Load), TDD=1.1523 (\%)

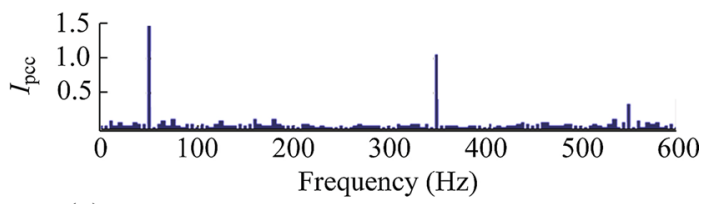

(c) $I_{\mathrm{pcc}}$ of ac system 3 (\% of Load), TDD=2.2817 (\%)

Fig. 5 Case 1: AC side phase current spectra

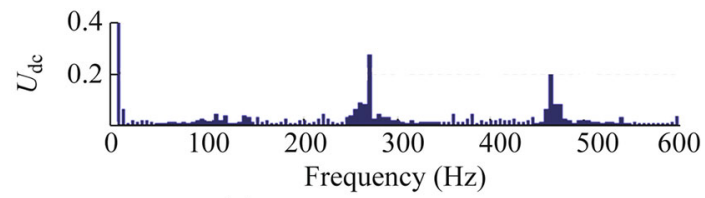

(a) $U_{\mathrm{dc}}\left(\%\right.$ of $\left.U_{\mathrm{dc} 0}\right)$ of $\mathrm{VSC} 1$

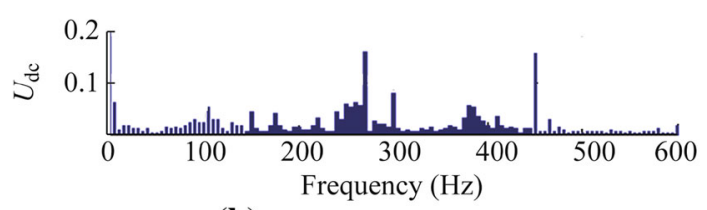

(b) $U_{\mathrm{dc}}\left(\%\right.$ of $\left.U_{\mathrm{dc} 0}\right)$ of $\mathrm{VSC} 2$

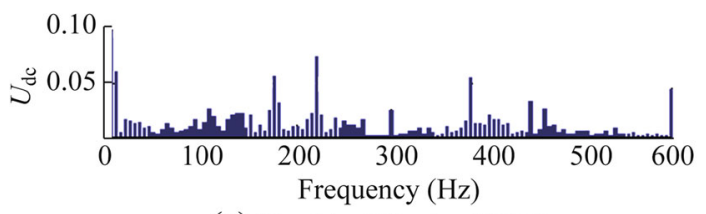

(c) $U_{\mathrm{dc}}\left(\%\right.$ of $\left.U_{\mathrm{dc} 0}\right)$ of $\mathrm{VSC} 3$

Fig. 6 Case 1: DC side voltage spectra

\subsection{Case 3: ac system with background harmonics}

Obviously, inter-harmonic transferred to the converter station from the connected ac system is one of the interharmonic sources of the multi-terminal VSC-based HVDC system. As can be seen from Fig. 4, the dc side of the converter Station 3 resonated at $135 \mathrm{~Hz}$ with larger amplitude than that at the same frequency at the converter Station 1 , while the dc side of the converter 2 resonated at $115 \mathrm{~Hz}$. The $135 \mathrm{~Hz}$ harmonic could be caused by positive sequence harmonic at frequency of $195 \mathrm{~Hz}$ from the ac system 1 , or at frequency of $185 \mathrm{~Hz}$ from ac systems 2 or 3 . The ac system 1 is assumed to be distorted with a $10 \%$ positive-sequence harmonic voltage at $195 \mathrm{~Hz}$ for the first investigation (called the Case $3 \mathrm{a}$ ). As a result, the dc-side voltage mainly distorted at $135 \mathrm{~Hz}$ which is fed back to the VSC1 ac side to produce a new harmonic at $75 \mathrm{~Hz}$. However, the ac side had no series resonance at $75 \mathrm{~Hz}$ and hence the feedback again to dc side is insignificant.

The dominant inter-harmonic, which is $135 \mathrm{~Hz}$, transferred to the remote converter Stations 2 and 3, and produced the same harmonic orders in their ac-side system but with different magnification and damping factors. Due to a parallel resonance at $135 \mathrm{~Hz}$ at dc terminal of the converter Station 3, the distortion in the ac system 3 is larger than that in the ac system 1 (Figs. 8, 9).

The second investigation on the production of interharmonics is that a background inter-harmonic at $185 \mathrm{~Hz}$ in the ac system 3 (called the Case $3 b$ ). The new inter-harmonics in all three ac systems are the same to the Case $3 \mathrm{a}$ but with smaller magnification despite of larger resonance

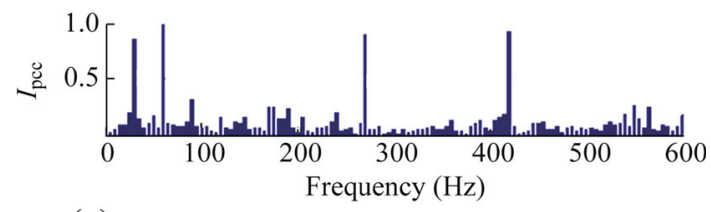

(a) $I_{\mathrm{pcc}}$ of ac system 1 (\% of Load), TDD=2.7729 (\%)

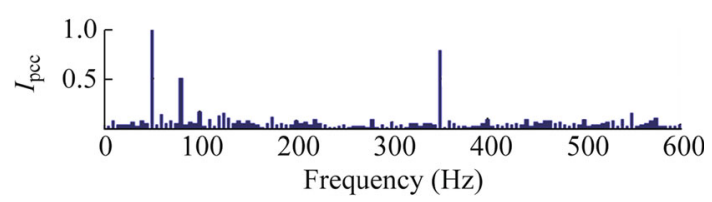

(b) $I_{\text {pcc }}$ of ac system $2(\%$ of Load), TDD $=1.2702(\%)$

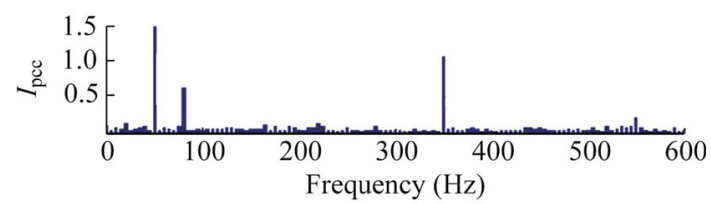

(c) $I_{\mathrm{pcc}}$ of ac system 3 (\% of Load), TDD=2.3147 (\%)

Fig. 7 AC current inter-harmonics caused by dc capacitors 


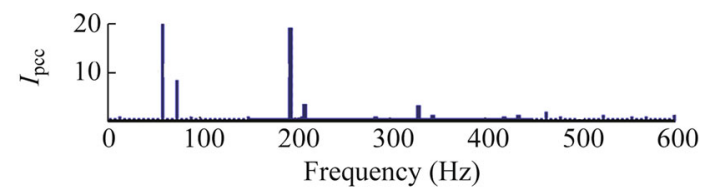

(a) $I_{\text {pcc }}$ of ac system 1 ( $\%$ of Load), TDD=30.3797 (\%)

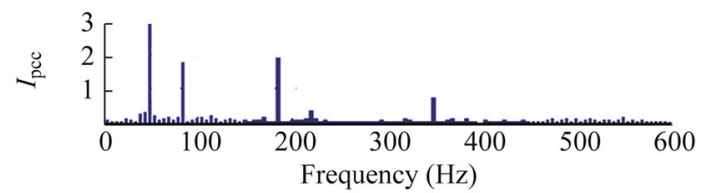

(b) $I_{\mathrm{pcc}}$ of ac system $2(\%$ of Load), TDD=3.2818 (\%)

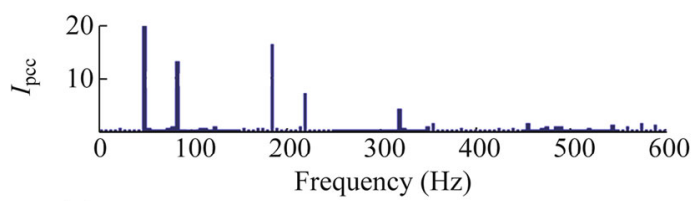

(c) $I_{\mathrm{pcc}}$ of ac system $3(\%$ of Load), TDD=36.0883 (\%)

Fig. 8 Case 3a: AC side current spectra

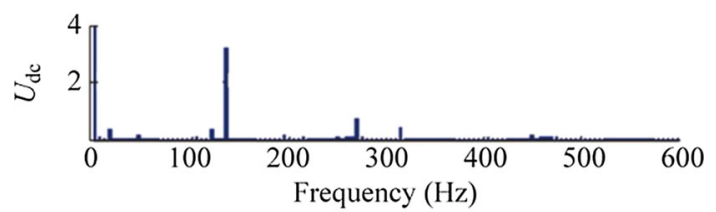

(a) $U_{\mathrm{dc}}\left(\%\right.$ of $\left.U_{\mathrm{dc} 0}\right)$ of $\mathrm{VSC} 1$

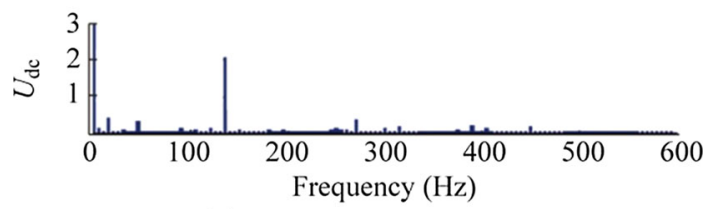

(b) $U_{\mathrm{dc}}\left(\%\right.$ of $\left.U_{\mathrm{dc} 0}\right)$ of VSC2

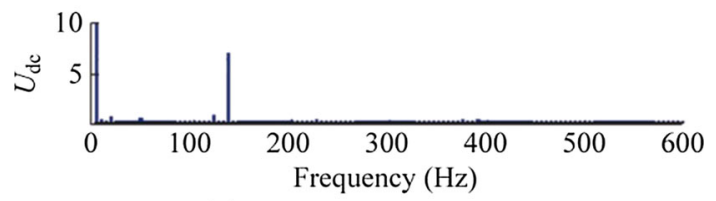

(c) $U_{\mathrm{dc}}\left(\%\right.$ of $\left.U_{\mathrm{dc} 0}\right)$ of $\mathrm{VSC} 3$

Fig. 9 Case 3a: DC side voltage spectra

magnitude at the dc terminal of the VSC3. This is because the power contribution of the VSC3 to the grid is smaller than that of the VSC1. The values of individual inter-harmonics and the total distortion are shown in Table 1.

Another case (called the Case 3c) is also investigated to illustrate the effect of resonance on the dc side on the interharmonic and harmonic distortion of the system. In this case the harmonics with the same magnitudes relative to the Case $3 \mathrm{a}$ and $3 \mathrm{~b}$ are injected into the system, which are the 2nd-order negative sequence harmonic in the ac system
1 and the 4th-order positive-sequence harmonic in the ac system 3. Although the harmonic source is doubled, because of no resonance appeared at relative harmonics on the dc side, the total distortions are smaller than the Case $3 \mathrm{a}$, which are $12.72 \%, 5.13 \%$, and $27.67 \%$ relative to the ac systems 1,2 , and 3 . It can be seen from the waveform of the current in these three cases in Fig. 10 where the blue line (Case 3a) is the most distorted.

\subsection{Case 4: series-parallel dc grid}

The series-parallel dc grid was configured by cutting out a dc link of the mesh dc grid. To investigate the harmonic characteristic of the system in this case, the dc link between the converter Stations 2 and 3 is out of service, while the ac system 1 is still injected a positive background inter-harmonic at $195 \mathrm{~Hz}$. As a result, the impedance-frequency characteristic of the dc grid is different, thus affecting the harmonic levels. It can be seen in Table 1, the harmonic demand distortions, as well as the significant additional harmonics are all smaller than those in the Case 3a above.

\subsection{Case 5: unsymmetrical fault in ac systems}

In this section, single phase-to-ground faults at the PCCs of the ac system 1 (called the Case 5a) and the ac system 3 (called the Case $5 b$ ) are investigated.

In the Case 5a, during the fault, a series of inter-harmonics are produced on the dc side as well as the ac side of either converter Stations 2 or 3 . The dc side of the converter Station 1 had a harmonic with frequency of $120 \mathrm{~Hz}$ due to the negative-sequence component of the ac side voltage. This $120 \mathrm{~Hz}$ harmonic is transferred to the converter Stations 2 and 3 acting as inter-harmonic sources, consequently causing inter-harmonic on the ac side of each converter with dominant at $170 \mathrm{~Hz}$ and $70 \mathrm{~Hz}$; the former is the positive sequence and the latter is the negative sequence, as shown in Fig. 11 and Table 2.

The same mechanism of inter-harmonic producing in the Case $5 \mathrm{~b}$ is the same to the Case $5 \mathrm{a}$ but with a $100 \mathrm{~Hz}$ harmonic on the dc side. The ac side of VSC2, therefore, has an additional positive sequence harmonic at $150 \mathrm{~Hz}$; the ac side of VSC1 has two dominant inter-harmonics at $160 \mathrm{~Hz}$ (positive sequence) and $40 \mathrm{~Hz}$ (negative sequence). Notably, the negative sequence inter-harmonic at $40 \mathrm{~Hz}$, which is smaller than the fundamental frequency, if a series resonance at this frequency occurs, a modest inter-harmonic voltage may drastically amplify the inter-harmonic current, damaging the rotating machine connected to the ac system 1 . The values of individual harmonics are shown in Table 2. The fault in the ac system 3 in this case has insignificant effect on the other ac systems. 
Table 1 Background inter-harmonic propagation from one ac system to the other ones through dc grid

\begin{tabular}{|c|c|c|c|c|c|c|c|c|c|}
\hline \multirow[t]{2}{*}{$f(\mathrm{~Hz})$} & \multicolumn{3}{|c|}{ Case $3 \mathrm{a}(\%$ of load) } & \multicolumn{3}{|c|}{ Case $3 b$ ( $\%$ of load) } & \multicolumn{3}{|c|}{ Case 4 (\% of load) } \\
\hline & $I_{\mathrm{pcc} 1}$ & $I_{\mathrm{pcc} 2}$ & $I_{\mathrm{pcc} 3}$ & $I_{\mathrm{pcc} 1}$ & $I_{\mathrm{pcc} 2}$ & $I_{\mathrm{pcc} 3}$ & $I_{\mathrm{pcc} 1}$ & $I_{\mathrm{pcc} 2}$ & $I_{\mathrm{pcc} 3}$ \\
\hline 75 & 7.61 & - & - & 2.99 & - & - & 11.06 & - & - \\
\hline 85 & - & 1.84 & 12.88 & - & 0.82 & 2.11 & - & 0.966 & 10.18 \\
\hline 185 & - & 1.95 & 16.00 & - & 0.79 & 15.68 & - & 0.97 & 12.88 \\
\hline 195 & 18.62 & - & - & 4.14 & - & - & 13.63 & - & - \\
\hline 210 & 2.92 & - & - & 0.43 & - & - & 5.66 & - & - \\
\hline 220 & - & 0.36 & 6.92 & - & 0.03 & 0.22 & - & 0.38 & 4.83 \\
\hline 320 & - & 0.09 & 3.95 & - & 0.05 & 0.28 & - & - & 2.676 \\
\hline 330 & 2.57 & - & - & 0.50 & - & - & 4.23 & - & - \\
\hline TDD & 30.28 & 3.42 & 36.57 & 8.09 & 1.76 & 26.80 & 28.88 & 2.15 & 28.84 \\
\hline
\end{tabular}

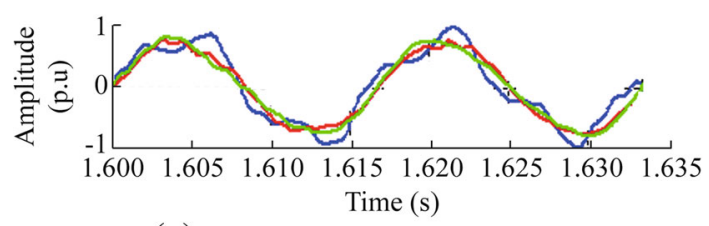

(a) The waveform of $I_{\mathrm{pcc}}$ of ac system 1

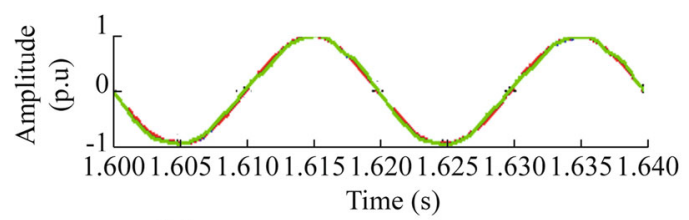

(b) The waveform of $I_{\text {pcc }}$ of ac system 2

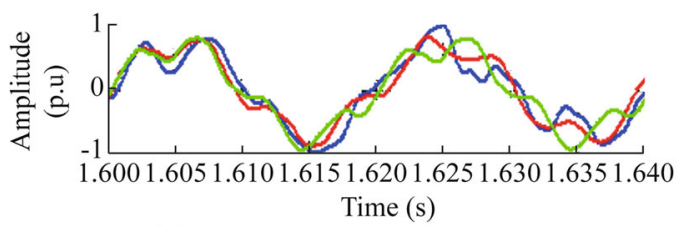

(c) The waveform of $I_{\text {pcc }}$ of ac system 3

- Case 3a; - Case 3b; - Case 3c

Fig. 10 AC side phase current waveform

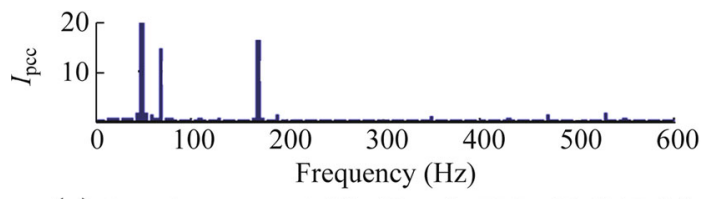

(a) $I_{\mathrm{pcc}}$ of ac system $2(\%$ of Load), TDD $=33.6337(\%)$

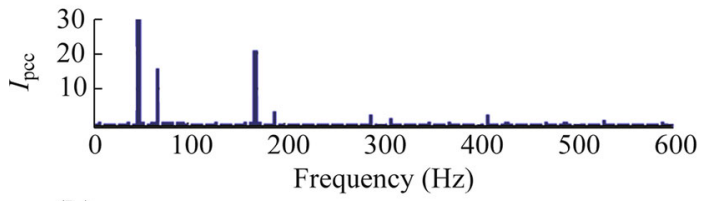

(b) $I_{\text {pcc }}$ of ac system 3 ( $\%$ of Load), TDD $=42.6982(\%)$

Fig. 11 Case 5a: fault at PCC1 causing inter-harmonics in the other ac systems
Table 2 Effects of unsymmetrical condition on harmonic distortion

\begin{tabular}{|c|c|c|c|c|}
\hline \multirow[t]{2}{*}{$f(\mathrm{~Hz})$} & \multicolumn{2}{|c|}{ Case $5 \mathrm{a}(\%$ of load) } & \multicolumn{2}{|c|}{ Case $5 b(\%$ of load $)$} \\
\hline & $I_{\mathrm{pcc} 2}$ & $I_{\mathrm{pcc} 3}$ & $I_{\mathrm{pcc} 1}$ & $I_{\mathrm{pcc} 2}$ \\
\hline 40 & - & - & 0.81 & - \\
\hline 70 & 14.03 & 15.57 & - & - \\
\hline 150 & - & - & - & 2.51 \\
\hline 160 & - & - & 1.26 & - \\
\hline 170 & 15.96 & 20.18 & - & - \\
\hline TDD & 33.63 & 42.70 & 2.90 & 3.10 \\
\hline
\end{tabular}

\section{Conclusion}

The inter-harmonics in multi-terminal VSC-based HVDC systems are originated from the characteristic of each converter in asynchronous connection, the distorting in the connected ac systems, and the unsymmetrical fault or unbalance.

In all cases, there are a series of inter-harmonics produced in the connected ac systems and the dc grid. For confirmation, the simulation models are established and implemented. Simulation results are in agreement with the theoretical analyses. The dc grid is a complex system with dc resonance points occurred at low frequency range, and the operating configurations also affect harmonic transfer through the grid. The harmonic and interharmonic interaction at each converter station depended on the characteristic of the converter its own is also affected by the interaction from the other converter stations. Corresponding to the simulation results, the effect of dc capacitors is significant in raising a series of lowfrequency inter-harmonics in the ac side systems. Moreover, the unsymmetrical ac system has significant effect on harmonic interaction in the multi-terminal VSC-based HVDC system, it is proportional to its power contribution. 
Acknowledgements This work was supported by "111" Project (No. B08013) of China, and Natural Science Foundation of China (No. 51177044).

Open Access This article is distributed under the terms of the Creative Commons Attribution 4.0 International License (http:// creativecommons.org/licenses/by/4.0/), which permits unrestricted use, distribution, and reproduction in any medium, provided you give appropriate credit to the original author(s) and the source, provide a link to the Creative Commons license, and indicate if changes were made.

\section{Appendix A}

\section{Switching function of VSC}

The switching function $k_{x}(t)$ of phase $x(x=\mathrm{a}, \mathrm{b}, \mathrm{c})$ of a three-phase three-level NPC VSC in time domain [4]:

$$
\begin{aligned}
k_{x}(t)= & M \cos \left(\omega_{1} t+\theta_{x}\right)+\frac{2}{\pi} \sum_{m=2,4,6 \ldots}^{\infty} \frac{1}{m} \sum_{n=-\infty}^{+\infty} \cdot \\
& {\left[\begin{array}{l}
J_{2 n+1}(m \pi M) \cos n \pi \\
\cdot \cos \left(m\left(\omega_{c} t+\delta\right)+[2 n+1]\left(\omega_{1} t+\theta_{x}\right)\right)
\end{array}\right]+\frac{8}{\pi^{2}} . } \\
& \sum_{m=1,3,5 \ldots}^{\infty} \frac{1}{m} \sum_{n=-\infty}^{+\infty}\left[\begin{array}{l}
J_{k m} \cos n \pi \\
\cdot \cos \left(m\left(\omega_{c} t+\delta\right)+2 n\left(\omega_{1} t+\theta_{x}\right)\right)
\end{array}\right]
\end{aligned}
$$

$J_{k m}=\sum_{k=1}^{\infty} J_{2 k-1}([2 m-1] \pi M) \frac{(2 k-1)}{(2 k+2 n-1)(2 k-2 n-1)}$

where $M$ is the modulation index; $\omega_{1}$ is the frequency of the modulation wave; $\omega_{c}$ is the frequency of the carrier wave; $m$ is the group index (multiple of switching frequency); $n$ is the side band harmonic index from each group; $\theta_{x}$ is the modulation wave phase shift for each phase $x ; \delta$ is the carrier wave phase shift; $J_{2 n+1}, J_{2 k-1}$ are the first kind Bessel functions.

Clearly, the switching function contains a fundamental frequency component and high frequency harmonic components. The even carrier side-band harmonics only exist around the odd carrier multiples ( $m$ is odd), and odd carrier side-band harmonics only exist around the even carrier multiples ( $m$ is even).

Applying space vector theory, the space vector representation form of the VSC's switching function is composed of three component sets as:

$\bar{K}(t)=\bar{K}_{1}(t)+\sum_{m=1}^{\infty} \sum_{n=-\infty}^{+\infty} \bar{K}_{m n}^{+}(t)+\sum_{m=1}^{\infty} \sum_{n=-\infty}^{+\infty} \bar{K}_{m n}^{-}(t)$ $\left\{\begin{aligned} \bar{K}_{1}(t) & =M \mathrm{e}^{\mathrm{j} \omega_{1} t} \\ \bar{K}_{m n}^{+}(t) & =\hat{K}_{m n}^{+} \mathrm{e}^{\mathrm{j} N_{m n}^{+} \omega_{1} t} \\ \bar{K}_{m n}^{-}(t) & =\hat{K}_{m n}^{-} \mathrm{e}^{-\mathrm{j} N_{m n}^{-} \omega_{1} t}\end{aligned}\right.$

where $\hat{K}_{m n}^{+}$and $\hat{K}_{m n}^{-}$are the amplitudes of the positive- and negative-sequence switching components; $N_{m n}^{+}=3 i+1$ and $N_{m n}^{-}=3 i-1$, where $i$ is an integer. The zero-sequence switching component with $N_{m n}^{0}=3 i$ does not appear in (A3).

\section{Space vector representation of harmonic}

For the three-phase harmonic quantity,

$\left\{\begin{array}{l}f_{h \mathrm{a}}(t)=\hat{f} \cos \left(h \omega_{1} t\right) \\ f_{h \mathrm{~b}}(t)=\hat{f} \cos \left(\frac{h \omega_{1} t-2 h \pi}{3}\right) \\ f_{h \mathrm{c}}(t)=\hat{f} \cos \left(\frac{h \omega_{1} t+2 h \pi}{3}\right)\end{array}\right.$

The space vector form in (A6) contains a positivesequence component (the first part), a negative-sequence component (the second part).

$$
\begin{gathered}
\bar{f}_{h}=\frac{\hat{f}}{3}\left[1+\mathrm{e}^{-\mathrm{j}(h-1) \frac{2 \pi}{3}}+\mathrm{e}^{-\mathrm{j}(h-1) \frac{4 \pi}{3}}\right] \mathrm{e}^{\mathrm{j} h \omega_{1} t}+ \\
\frac{\hat{f}}{3}\left[1+\mathrm{e}^{\mathrm{j}(h+1) \frac{2 \pi}{3}}+\mathrm{e}^{\mathrm{j}(h+1) \frac{4 \pi}{3}}\right] \mathrm{e}^{-\mathrm{j} h \omega_{1} t}
\end{gathered}
$$

\section{References}

[1] Flourentzou N, Agelidis VG, Demetriades GD (2009) VSCbased HVDC power transmission systems: an overview. IEEE Trans Power Electron 24(3):592-602

[2] Van Hertem D, Ghandhari M (2010) Multi-terminal VSC HVDC for the European supergrid: obstacles. Renew Sustain Energy Rev 14(9):3156-3163

[3] Ahmed N, Haider A, Van Hertem D et al (2011) Prospects and challenges of future HVDC SuperGrids with modular multilevel converters. In: Proceedings of the 14th European conference on power electronics and applications (EPE'11), Birmingham, 30 August-1 September 2011, $10 \mathrm{pp}$

[4] Holmes D, Lipo T (2003) Pulse width modulation for power converters: principles and practice. IEEE Press, Piscataway

[5] Jiang Y, Ekstrom A (1997) General analysis of harmonic transfer through converters. IEEE Trans Power Electron 12(2): 287-293

[6] Tang LX, Ooi BT (2001) Converter nonintegral harmonics from AC network resonating with DC network. In: Proceedings of the 26th industry applications society annual meeting, vol 4, Chicago, 30 September-4 October 2001, pp 2186-2192

[7] Gunther EW (2001) Interharmonics in power systems. In: Proceedings of the IEEE power engineering society summer meeting, vol 2, Vancouver, 15-19 July 2001, 6 pp

[8] Testa A, Akram MF, Burch R et al (2007) Interharmonics: theory and modeling. IEEE Trans Power Deliv 22(4):2335-2348 
[9] Nguyen P, Han M (2014) Study on harmonic propagation of VSC-based HVDC systems. In: Proceedings of the 2014 international conference on power system technology (POWERCON'14), Chengdu, 20-22 October 2014, pp 2146-2153

[10] Shore NL, Adamson K, Bard P et al (1996) DC side filters for multiterminal HVDC systems. IEEE Trans Power Deliv 11(4):1970-1984

[11] Yazdani A, Iravani R (2010) Voltage sourced converters in power systems: modeling, control and applications. IEEE Press, Hoboken

[12] Gopalakrishnan KS, Narayanan G (2013) Harmonic analysis of DC-link capacitor current in sinusoidally modulated neutralpoint-clamped inverter. In: Proceedings of the national power electronics conference, Kanpur, 20-22 December 2013, 6 pp

[13] Zhang GB, Xu Z, Wang GZ (2001) Control strategy for unsymmetrical operation of HVDC-VSC based on the improved instantaneous reactive power theory. In: Proceedings of the 7th international conference on AC-DC power transmission, London, 28-30 November 2001, pp 262-267

[14] IEEE Std 519-2014 (2014) IEEE recommended practice and requirements for harmonic control in electric power systems

Minxiao HAN was born in Shannxi, China, in 1963. He received his B.Sc. from Xi' an Jiaotong University in 1984, M.Sc. and Ph.D. from
North China Electric Power University (NCEPU) in 1987 and 1995 respectively. He was a visiting research fellow in Queen's University of Belfast, U.K. and post-doctoral research fellow with Kobe University, Japan. He is active in professional society activities and international cooperation relating with the field of application of power electronics in power system including HVDC \& FACTS, power quality and the integration of renewable generation in power network.

Phuchuy NGUYEN received his B.Sc and M.Sc degree from Hanoi University of Science and Technology (HUST), Viet Nam in 2003 and 2010 respectively. Now he is a Ph.D. student in the Electric Power System and Its Automation at North China Electric Power University (NCEPU), Beijing, China. The main research interest is in the field of flexible HVDC transmission systems and power quality.

Wenli YAN is Professor of North China Electric Power University (NCEPU), received her B.Sc. from Sichuan University, China in 1987 and M.Sc. from Beijing Normal University in 1990, both with the field of mathematics. Her research interests mainly include the stochastic system analysis and numerical calculation. 